\title{
Mitral valve repair rates correlate with surgeon and institutional experience
}

Damien J. LaPar, MD, MSc, ${ }^{\mathrm{a}}$ Gorav Ailawadi, MD, ${ }^{\mathrm{a}} \mathrm{James}$ M. Isbell, MD, MSCI, ${ }^{\mathrm{a}}$ Ivan K. Crosby, MD, ${ }^{\mathrm{a}}$ John A. Kern, MD, ${ }^{\mathrm{a}}$ Jeffrey B. Rich, MD, ${ }^{\mathrm{b}}$ Alan M. Speir, MD, ${ }^{\mathrm{c}}$ and Irving L. Kron, MD, ${ }^{\mathrm{a}}$ Investigators for the Virginia Cardiac Surgery Quality Initiative

Objectives: Mitral valve (MV) repair rates have lagged despite reported superior outcomes in patients with mitral regurgitation. The purpose of the present study was to evaluate the relationship between procedure volume and the propensity for MV repair in a multi-institution, regional patient cohort.

Methods: Society of Thoracic Surgeons-certified patient records of those undergoing MV repair or MV replacement (MVR) for moderate or severe mitral regurgitation were evaluated from 17 different centers (2001-2011). The relationship between the annual hospital and surgeon volume and the propensity for MV repair over MVR was analyzed using multivariable, mortality risk-adjusted models with restricted cubic splines.

Results: A total of 4194 patients were evaluated (MV repair, 2516; MVR, 1662). The median annual mitral procedure volume was 54 operations for hospitals and 13 operations for surgeons. The overall MV repair rate was $60 \%$, with significant variations among hospitals (range, 35\%-70\%) and surgeons (range, 0\%-90\%). The MVR patients presented with higher Society of Thoracic Surgeons Predicted Risk of Mortality scores $(6 \%$ vs $2 \%, P<.001)$. After adjustment for Predicted Risk of Mortality score, both annual hospital $(P=.04)$ and surgeon $(P<.0001)$ procedure volume were associated with the probability of MV repair. The likelihood for MV repair correlated with an operative volume of $\geq 20$ procedures annually. Among surgeons and hospitals performing $\geq 20$ mitral operations annually, MV repair rates were greater $(73 \%$ vs $26 \%$ and $62 \%$ vs $37 \%$, respectively, $P<.001$ for both).

Conclusions: Significant variation in the performance of MV repair over MVR for mitral regurgitation persists in the modern surgical era. Average annual surgeon volume was more significantly associated with MV repair rate than institutional volume, with an increased likelihood for performance of MV repair among surgeons performing $>20$ procedures annually. In the upcoming era of percutaneous MV repair, surgeon volume and expertise as a gatekeeper should dictate access to this technology and the decisions for the best approach to MV repair. (J Thorac Cardiovasc Surg 2014;148:995-1004)

Mitral valve (MV) surgery remains the standard of care for severe mitral regurgitation (MR) (grade 3-4+). The current joint American College of Cardiology/American Heart Association and European Society of Cardiology guidelines reflect the clear benefit of MV surgery over medical management for severe MR. ${ }^{1,2}$ Despite these recommendations, only $50 \%$ of patients meeting the American College of Cardiology/American Heart Association guidelines for surgical treatment undergo surgery. ${ }^{3,4}$ Moreover, significant variation in the performance of MV repair or

From the Department of Surgery, ${ }^{\mathrm{a}}$ University of Virginia, Charlottesville, Va; Sentara Heart Hospital, ${ }^{\mathrm{b}}$ Norfolk, Va; and Inova Heart and Vascular Institute, ${ }^{\mathrm{c}}$ Falls Church, Va.

Disclosures: Authors have nothing to disclose with regard to commercial support.

Read at the 94th Annual Meeting of The American Association for Thoracic Surgery, Toronto, Ontario, Canada, April 26-30, 2014.

Received for publication April 30, 2014; revisions received June 16, 2014; accepted for publication June 25, 2014; available ahead of print July 18, 2014.

Address for reprints: Irving L. Kron, MD, Department of Surgery, University of Virginia, PO Box 800679, Charlottesville, VA 22908 (E-mail: ilk@virginia.edu). $0022-5223 / \$ 36.00$

Copyright (C) 2014 by The American Association for Thoracic Surgery http://dx.doi.org/10.1016/j.jtcvs.2014.06.039
MV replacement (MVR) for moderate to severe MR has been demonstrated among hospitals and surgeons. ${ }^{5}$ Although the accumulated data have favored reduced operative mortality and morbidity for MV repair over MVR, including the recently published Cardiothoracic Surgery Trials Network Severe Ischemic Mitral Regurgitation randomized controlled trial, ${ }^{6}$ the overall performance of MV repair appears underused. Recent nationwide estimates have suggested usage of MV repair for functional MR of approximately $40 \%{ }^{7}$ Although a few noteworthy reports have been published describing both patient-level and surgeon-related factors associated with the performance of MV repair, ${ }^{5,7,8}$ additional multi-institution analyses using rigorous risk adjustment modeling methods are warranted to further characterize the individual influence of hospital and surgeon mitral procedure volume on the propensity for MV treatment allocation.

The purpose of the present study was to evaluate the relationship between annual surgeon and hospital procedure volume and the likelihood for MV repair in a multi-institution, regional cohort of patients with moderate 


$$
\begin{aligned}
& \text { Abbreviations and Acronyms } \\
& \text { ACSD }=\text { Adult Cardiac Surgery Database } \\
& \text { MR }=\text { mitral regurgitation } \\
& \text { MV }=\text { mitral valve } \\
& \text { MVR }=\text { mitral valve replacement } \\
& \text { PROM }=\text { Predicted Risk of Mortality } \\
& \text { RCS }=\text { restricted cubic spline } \\
& \text { STS }=\text { Society of Thoracic Surgeons } \\
& \text { VCSQI }=\text { Virginia Cardiac Surgery Quality } \\
& \text { Initiative }
\end{aligned}
$$

to severe MR. We hypothesized that significant variations in MV repair rates would be present among surgeons and hospitals in the modern surgical era; the probability for performance of MV repair over MVR would correlate with the average annual surgeon and/or hospital volume; and the effect of surgeon and hospital volume on the propensity for performance of MV repair over MVR would be independent of the effects of preoperative patient risk.

\section{METHODS}

The Virginia Cardiac Surgery Quality Initiative (VCSQI) consists of 17 cardiac surgical centers within the Commonwealth of Virginia that hold regular meetings to exchange and compare de-identified patient information to improve cardiac surgical care, quality, and costs. VCSQI centers perform approximately $99 \%$ of the Commonwealth's cardiac operations, contributing patient data to the Society of Thoracic Surgeons (STS) Adult Cardiac Surgery Database (ACSD).

The present study was exempt from formal institutional review board review at each participating hospital owing to the secondary analysis of the VCSQI de-identified (absence of Health Insurance Portability and Accountability Act patient identifiers) data registry and because the data were collected for quality analysis and purposes other than research.

\section{Patients and Data Acquisition}

De-identified patient data were extracted from the VCSQI data registry for the study period (January 1, 2001 to December 31, 2011). All records included patients undergoing isolated MV repair or MVR for moderate or severe MR (STS procedure type "MV repair" and "MV replacement"). Patients undergoing concomitant coronary artery bypass grafting, other valve procedures, or arrhythmia ablation procedures were excluded. All mitral procedures used standard open surgical approaches to MV surgery with the use of cardiopulmonary bypass support. Patient preoperative risk was assessed by the prevalence of patient comorbid disease, the degree of MR, operative status, and individually calculated STS Predicted Risk of Mortality (PROM) scores.

\section{Measured Outcomes}

The primary outcomes of interest included the risk-adjusted association between the probability of MV repair and average annual surgeon and hospital procedure volume. The secondary outcomes of interest included variations in MV repair rates and mitral procedure volume among surgeons and hospitals and the identification of a threshold volume at which MV repair was more commonly performed over MVR, independent of the baseline patient risk. The average annual procedure volume was calculated for each surgeon and hospital according to the performance of operations throughout the entire study period. Standard STS clinical definitions for all analyzed variables were used. ${ }^{9}$

\section{Statistical Analysis}

Descriptive statistics. All statistical analyses were designed to test the null hypotheses that the average annual procedure volume would not be significantly associated with the likelihood for MV repair (vs MVR). All study outcomes and data comparisons were established a priori before data collection. Categorical variables are expressed as group percentages and continuous variables as either the mean \pm standard deviation or median and 25th, 75th percentile, depending on the overall variable distribution. Univariate comparisons included either Pearson's chi-square or Fisher's exact test for categorical variables and either independent-sample, single-factor, analysis of variance for normally distributed data or the Wilcoxon rank sum test for non-normally distributed data. Two-sided $P$ values were used to define variable associations.

Risk-adjusted regression models. Two separate multiple regression models were first used to estimate the confounder-adjusted associations between the probability of MV repair (vs MVR) and average annual hospital (model 1) and surgeon (model 2) procedure volume for patients undergoing isolated MV operations for moderate to severe MR. The average annual procedure volumes for both individual surgeons and hospitals were analyzed as continuous functions, using restricted cubic spline (RCS) smoothing transformations to account for both linear and nonlinear associations with the performance of MV repair. RCS functions are beneficial, because they use all data points to estimate the shape of the relationship between an exposure (STS PROM) and an outcome (operative mortality). The use of RCS transformations, therefore, provides a more robust method to determine whether nonlinear relationships exist between a continuous variable and a dependent outcome. The use of RCS forces the tails of a function to be linear, which simplifies the representation. For RCS functions, procedures volumes were analyzed using a total of 3 knots placed at the 5th, 50th, and 95th percentiles to define the tails of each function. The predicted association between procedure volume and MV repair were adjusted for the confounding effects of the preoperative patient risk profile (STS PROM) and the effects of operations performed by different surgeons (model 1) and at different hospitals (model 2) through the inclusion of these variables as model covariates. The relative strength of the association between the average annual procedure volume and probability for MV repair (vs MVR) compared with other modeled factors was determined by each factors' likelihood ratio (Wald chi-square statistic) within the model. In addition, the risk-adjusted RCS function for both average annual surgeon and hospital procedure versus the probability of MV repair was graphically represented to identify an apparent threshold value (or inflection point) for procedure volume that correlated with an increase in the likelihood for MV repair.

Risk-adjusted propensity matched analyses. To further verify the multivariable model results for the estimated effects of the average annual surgeon and hospital mitral procedure volume on the probability of MV repair, additional risk-adjusted propensity scorematched analyses were performed to compare the study cohorts of matched MV repair and MVR. Propensity scores were estimated using logistic regression modeling with performance of MV repair (vs MVR) as the response variable and patient age, sex, operative year, surgeon, hospital, operative status (elective, urgent, or emergency), operative incidence (first operation or reoperation), and a history of endocarditis or heart failure as possible confounding predictor variables. Propensity scores were then used to match MV repair and MVR patients in a 1:1 ratio using the nearest neighbor greedy method, resulting in equal-size study cohorts. The MV repair and MVR rates were then compared as a function of the estimated volume thresholds and adjusted relationship between the procedure volume, and likelihood for MV repair was modeled within this cohort. 
Model performance. All regression model performance was assessed using the $\mathrm{C}$ statistic and the Nagelkerke pseudo- $R^{2}$ statistic. The $\mathrm{C}$ statistic is equivalent to the area under the receiver operating characteristic curve for models with a dichotomous response variable. It provides an estimate of the model's ability to discriminate between observed instances of MV repair versus MVR. The Nagelkerke pseudo- $R^{2}$ statistic is a log-likelihood ratio chi-square-based measure analogous to the $R^{2}$ statistic in ordinary multiple regression. The Nagelkerke pseudo- $R^{2}$ statistic theoretically changes from 0 for models that provide no predictive information to 1 for models that predict perfectly and describes the degree of variance in the response variable (MV repair) explained by the model. All statistical analyses were conducted using R statistical software, version 3.0.2 (available at: http://www.R-project.org).

\section{RESULTS}

\section{Patient Characteristics and Operative Features for MV Operations}

A total of 4194 patients underwent MV procedures for moderate to severe MR during the study period, including $2516(60 \%)$ MV repair and $1662(40 \%)$ MVR operations. The descriptive statistics and frequencies of patient risk factors are listed in Table 1. Overall, patients undergoing MVR presented with greater preoperative risk (STS PROM, 5.6\% vs $1.7 \%, P<.0001$ ). Comorbid disease was more prevalent among the MVR patients, including cerebrovascular disease, chronic lung disease, hypertension $(P=.02)$, history of endocarditis, previous stroke $(P=.002)$, renal failure and hemodialysis, arrhythmia, heart failure, and advanced New York Heart Association class $(P<.001$ for all others). In addition, the MVR patients more commonly underwent a greater proportion of cardiac reoperations and urgent or emergency operations $(P<.001)$. Severe MR was also slightly more common among patients undergoing MVR $(81.6 \%$ vs $73.6 \%, P<.0001)$.

\section{Distribution of Hospital and Surgeon Volumes and MV Repair and MVR Rates}

Significant variation in hospital and surgeon MV procedure volumes and rates of MV repair were observed throughout the study period. The median annual mitral procedure volume was 54 operations annually for hospitals and 13 operations annually for surgeons. Figure 1 displays the distribution of the median annual MV procedure volume for hospitals (Figure 1,A) and surgeons (Figure 1, B). As expected, both hospital and surgeon volumes were not normally distributed. Moreover, the overall MV repair rate was $60 \%$, with significant variations among the hospitals (range, $35 \%-70 \% ; P<.0001$; Figure 2) and surgeons (range, $0 \%-90 \% ; P<.0001$; Figure 3 ).

\section{Adjusted Relationship Between Probability of MV Repair and MV Procedure Volume}

To determine the risk-adjusted relationship between the annual hospital and surgeon MV procedure volumes and the likelihood of MV repair, logistic regression modeling was performed. Considering the non-normal distribution
TABLE 1. Frequency of preoperative patient risk factors for patients undergoing mitral valve repair and replacement

\begin{tabular}{|c|c|c|c|}
\hline Risk factor & $\begin{array}{c}\text { MVR } \\
(n=1662)\end{array}$ & $\begin{array}{l}\text { MV repair } \\
(n=2516)\end{array}$ & $\begin{array}{c}P \\
\text { value }\end{array}$ \\
\hline Patient age (y) & $63.98 \pm 13.09$ & $61.94 \pm 11.84$ & $<.0001$ \\
\hline \multicolumn{4}{|l|}{ Sex } \\
\hline Female & 53.20 & 38.50 & $<.0001$ \\
\hline Male & 46.80 & 61.50 & \\
\hline Cerebrovascular disease & 16.10 & 9.60 & $<.0001$ \\
\hline Chronic lung disease & & & $<.0001$ \\
\hline None & 74.80 & 83.80 & \\
\hline Mild & 12.30 & 9.30 & \\
\hline Moderate & 7.90 & 4.80 & \\
\hline Severe & 5.00 & 2.10 & \\
\hline Diabetes & 25.40 & 23.40 & .15 \\
\hline Dyslipidemia & 67.10 & 67.40 & .86 \\
\hline Hypertension & 67.00 & 63.50 & .02 \\
\hline History of infective endocarditis & 12.70 & 4.30 & $<.0001$ \\
\hline Previous stroke & 7.80 & 5.40 & .002 \\
\hline Renal failure & 7.20 & 4.20 & $<.0001$ \\
\hline Renal failure (dialysis) & 6.50 & 3.00 & $<.0001$ \\
\hline Previous CABG & 9.50 & 4.50 & $<.0001$ \\
\hline Previous valve operation & 14.90 & 1.10 & $<.0001$ \\
\hline Arrhythmia & 27.50 & 14.40 & $<.0001$ \\
\hline NYHA classification & & & $<.0001$ \\
\hline I & 6.80 & 10.20 & \\
\hline II & 18.70 & 24.10 & \\
\hline III & 37.30 & 31.40 & \\
\hline IV & 23.30 & 15.10 & \\
\hline Unknown & 14.00 & 19.40 & \\
\hline Heart failure within $2 \mathrm{wk}$ & 58.70 & 44.80 & $<.0001$ \\
\hline Status & & & $<.0001$ \\
\hline Elective & 57.20 & 69.40 & \\
\hline Urgent & 37.00 & 29.30 & \\
\hline Emergency & 5.80 & 1.40 & \\
\hline Incidence & & & $<.0001$ \\
\hline First operation & 78.30 & 94.30 & \\
\hline Reoperation 1 & 19.40 & 5.40 & \\
\hline Reoperation 2 & 1.70 & 0.20 & \\
\hline Reoperation 3 & 0.50 & 0.00 & \\
\hline Reoperation $4+$ & 0.00 & 0.10 & \\
\hline Mitral valve insufficiency & & & $<.0001$ \\
\hline Moderate & 18.40 & 26.40 & \\
\hline Severe & 81.60 & 73.60 & \\
\hline STS PROM (\%) & $5.6(2.6,11.6)$ & $1.7(0.5,4.3)$ & $<.0001$ \\
\hline
\end{tabular}

Data presented as mean \pm standard deviation, $\%$, or median (25th, 75 th percentile). $M V R$, Mitral valve replacement; $M V$, mitral valve; $C A B G$, coronary artery bypass grafting; NYHA, New York Heart Association; STS, Society of Thoracic Surgeons; PROM, Predicted Risk of Mortality.

of each volume measure, the likelihood for MV repair was modeled as a function of both annual hospital (model 1) and surgeon (model 2) procedure volume with restricted cubic spline transformations as both linear and nonlinear terms (Table 2). The estimated associations were adjusted for the confounding influence of preoperative patient risk using STS PROM (with RCS transformations) and the influence of the individual surgeon (model 1) or hospital 

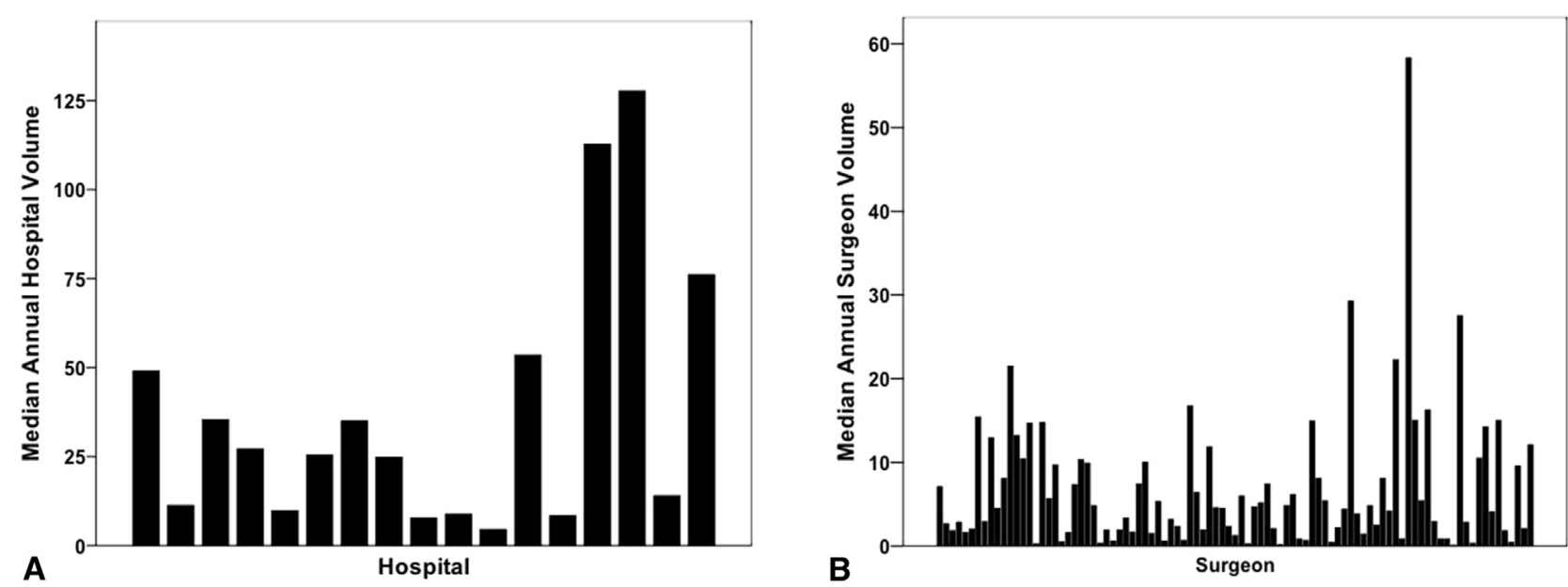

FIGURE 1. Histograms displaying the distribution of median annual mitral valve procedure volume for (A) hospitals and (B) surgeons.

(model 2). Thus, the associations between both annual hospital $(P=.03)$ and surgeon $(P<.0001) \mathrm{MV}$ procedure volume and the propensity for MV repair were significant. Annual surgeon volume demonstrated a much greater strength of association with the propensity for MV repair (likelihood ratio, 59.89). More importantly, the adjusted spline function revealed that an annual surgeon volume of $>20$ operations/y was associated with an increasing probability of MV repair, with dramatically increasing probabilities for surgeons performing $>20$ operations/y within both the entire patient cohort (Figure 4, $A$ ) and the propensity matched patient cohort (Figure $4, B$ ).

The statistical performance of this regression models achieved adequate discrimination, with a $\mathrm{C}$ statistic of 0.78 and 0.81 . The models explained $25 \%$ and $31 \%$ of the variance in the $\mathrm{MV}$ repair rates, as reflected by a Nagelkerke pseudo- $R^{2}$ value of 0.25 and 0.28 , respectively.

\section{Comparison of MV Repair as a Function of Procedure Volume Threshold}

After empirically testing the adjusted relationship between the performance of $\mathrm{MV}$ repair and annual procedure volume, a threshold value of $>20$ operations/y was identified and further evaluated to determine the effect of MV procedure choice. Overall, among surgeons and hospitals performing $\geq 20$ mitral operations annually, the MV repair rates were greater $(73 \%$ vs $26 \%$ and $62 \%$ vs $37 \%$, respectively; $P<.001$, for both).

To further validate the estimated associations described by models 1 and 2, we examined the rates of MV repair with respect to the observed threshold of $>20$ operations

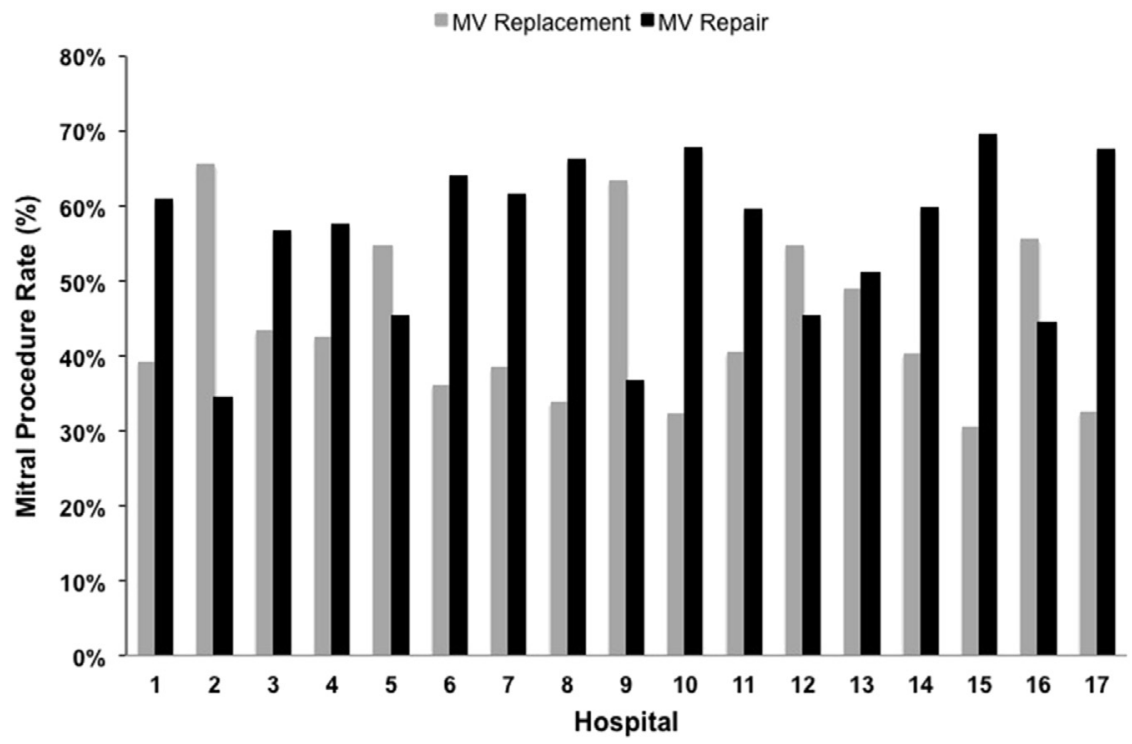

FIGURE 2. Comparison of hospital variation in mitral valve procedure rates. $M V$, Mitral valve. 


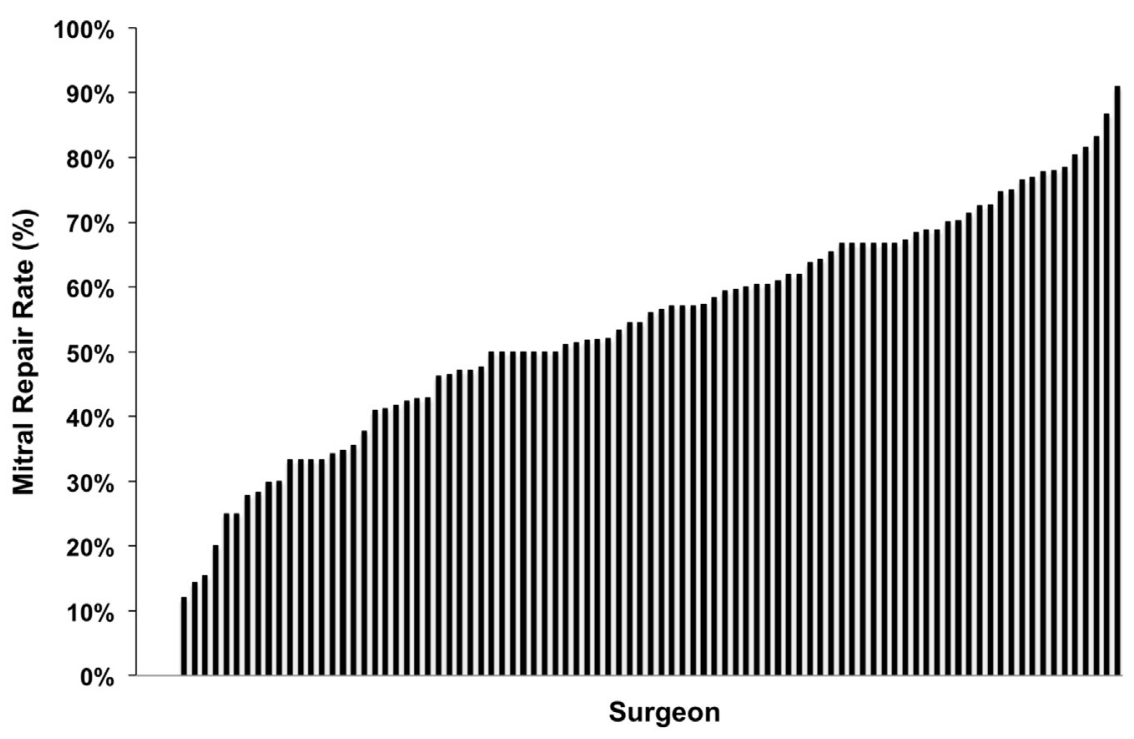

FIGURE 3. Variation in surgeon mitral valve repair rates.

annually in a 1:1 propensity matched cohort of $3322 \mathrm{MV}$ repair $(n=1661)$ and MVR $(n=1661)$ patients. Similar observations were noted in the propensity matched cohort of patients, with significantly greater rates of $\mathrm{MV}$ repair for hospitals and surgeons performing $>20$ operations annually (53\% vs $47 \%$ and $67 \%$ vs $39 \%$, respectively, $P<.0001$ for both).

\section{Comparison of Postoperative Outcomes for MV Repair and MVR}

The incidence of postoperative outcomes and hospital length of stay for propensity matched MV repair versus

TABLE 2. Multivariable regression results for the modeled outcome of likelihood of mitral valve repair (vs replacement) and its association with the influence of annual hospital (model 1) and surgeon (model 2) mitral procedure volume

\begin{tabular}{lccc}
\hline \multicolumn{1}{c}{ Factor } & $\begin{array}{c}\text { Likelihood } \\
\text { ratio (Wald } \\
\text { statistic) }\end{array}$ & AOR $(\mathbf{9 5} \%$ CI) & $\begin{array}{c}\boldsymbol{P} \\
\text { value }\end{array}$ \\
\hline $\begin{array}{c}\text { Model 1 } \\
\text { Annual hospital volume } \\
\quad \text { (nonlinear)* }\end{array}$ & 4.32 & $1.23(1.02-1.48)$ & .03 \\
$\begin{array}{l}\text { PROM (nonlinear)* } \\
\text { Surgeon }\end{array}$ & 96.05 & $0.15(0.12-0.18)$ & $<.0001$ \\
$\begin{array}{l}\text { Model 2 } \\
\text { Annual surgeon volume } \\
\quad \text { (nonlinear)* }\end{array}$ & 0.39 & $0.95(0.80-1.13)$ & .53 \\
$\begin{array}{l}\text { PROM (nonlinear) } \\
\text { Hospital }\end{array}$ & 59.89 & $1.25(1.04-1.72)$ & $<.0001$ \\
\hline
\end{tabular}

Each model adjusted influence of all covariate factors; model 1 performance characteristics, C-statistic, 0.78; Nagelkerke pseudo- $R^{2}=0.25$; model 2 performance characteristics, C-statistic, 0.81; Nagelkerke pseudo- $R^{2}=0.31$. AOR, Adjusted odds ratio; $C I$, confidence interval; PROM, Predicted Risk of Mortality. *Transformation of continuous variable using restricted cubic spline piecewise polynomial smoothing functions ( 3 knots at 5 th, 50th, and 95 th percentiles).
MVR patient populations are listed in Table 3. Patients undergoing MV repair were associated with lower rates of postoperative morbidity and 30-day and operative mortality.

\section{DISCUSSION}

The present study reports on the performance of MV operations in a statewide patient population with moderate to severe MR. The reported findings address the fundamental question related to the risk-adjusted influence of hospital and surgeon volume and/or experience on the likelihood for performance of MV repair. The present investigation has demonstrated that significant variation exists in the modern surgical era in the performance of MV repair at both the hospital and the individual surgeon level. These differences also appeared to be more strongly related to individual surgeon factors. Thus, with emerging percutaneous techniques for mitral repair, these data have demonstrated the critical role of the surgeon in the procedure choice for MR.

The $60 \%$ rate of the performance of MV repair in the present series demonstrated an improvement compared with previous reports. Previous single-institution and nationwide analyses have suggested an underusage of surgical MV repair in the setting of reported superior perioperative outcomes compared with MVR, including preservation of the left ventricular function, freedom from anticoagulation therapy, and lower early and late mortality. $5,7,8,10-12$ A clinically important estimate of nationwide rates of MV repair were provided by separate analyses of the STS ACSD by Gammie and colleagues ${ }^{5}$ and Bolling and colleagues, ${ }^{7}$ which demonstrated $\mathrm{MV}$ repair rates of $42 \%$ to $61 \%$ (from 2000 to 2007 ) and $41 \%$, respectively. 

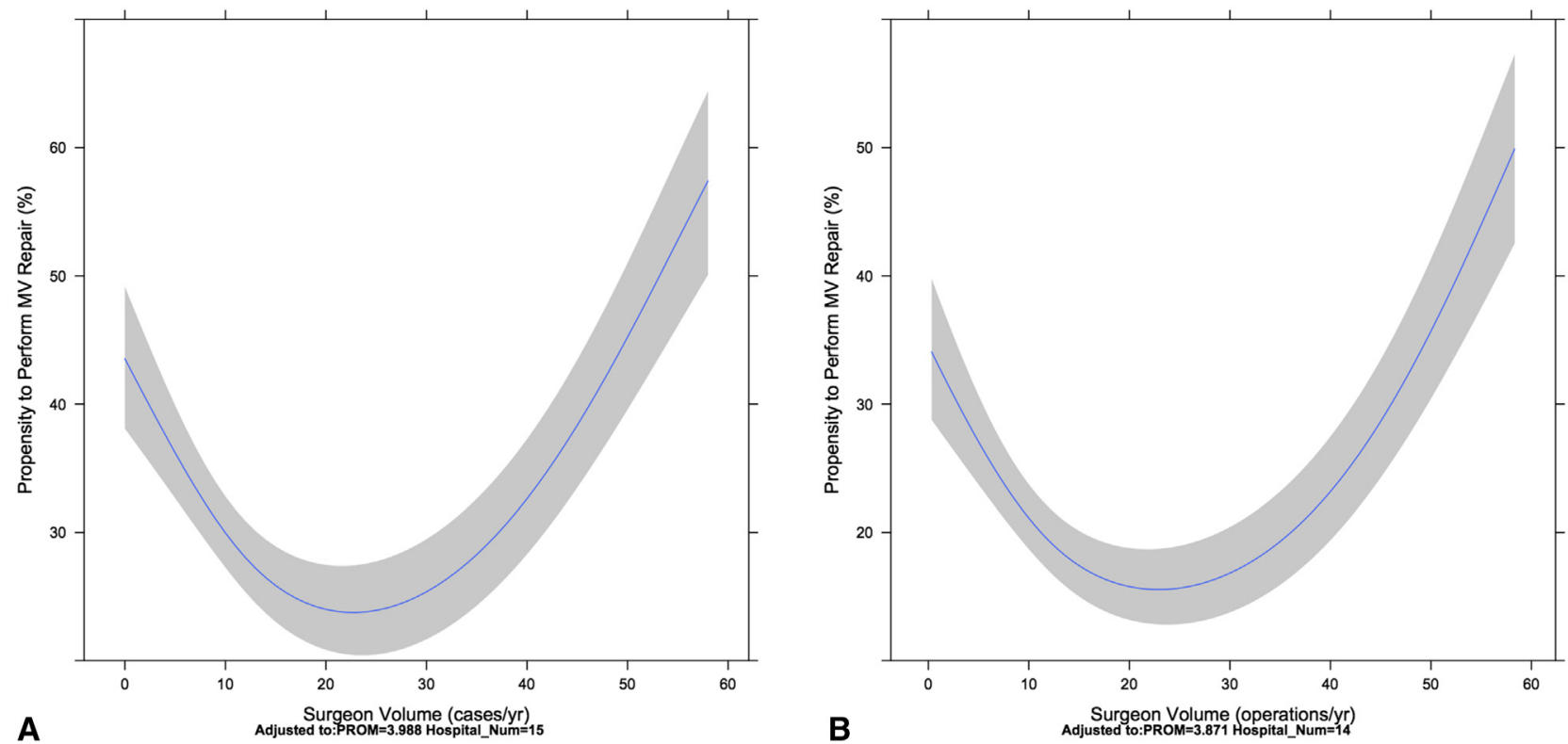

FIGURE 4. Plot of adjusted association between probability of mitral valve repair and the adjusted restricted cubic spline function for average annual surgeon mitral procedure volume within (A) the entire patient cohort and (B) a propensity matched patient group. MV, Mitral valve; PROM, Predicted Risk of Mortality.

These reports and the present analysis have demonstrated an overall improvement compared with the $38 \%$ mitral repair rate reported in the United States from 1999 to $2000 .^{12}$ Furthermore, the present study has demonstrated important variations in MV repair rates across hospitals, with MV repair rates ranging from $38 \%$ to $70 \%$, and among surgeons. Some surgeons exclusively performed MVR (ie, $0 \% \mathrm{MV}$ repair rate), and others performed MV repair in $90 \%$ of cases. These observations have potentially important clinical implications related to the influence of underlying mitral etiology and how such differences might affect referral for repair or replacement and individual surgeon MV procedure choice. Thus, 1 consideration in this and many analyses of MV operations using currently

TABLE 3. Postoperative outcomes for propensity matched patients undergoing mitral valve repair versus replacement

\begin{tabular}{lccc}
\hline \multicolumn{1}{c}{ Outcome } & $\begin{array}{c}\text { MV repair } \\
(\mathbf{n}=\mathbf{~ 1 6 6 1 )}\end{array}$ & $\begin{array}{c}\text { MVR } \\
(\mathbf{n}=\mathbf{1 6 6 1})\end{array}$ & $\boldsymbol{P}$ value \\
\hline Postoperative LOS (d) & $6(4,8)$ & $8(6,8)$ & $<.001$ \\
Myocardial infarction & 0.4 & 0.5 & .59 \\
Stroke & 1.3 & 2.3 & .04 \\
New-onset atrial fibrillation & 22.2 & 23.2 & .48 \\
Pneumonia & 2.6 & 6.9 & $<.001$ \\
Renal failure & 4.5 & 9.6 & $<.001$ \\
New-onset hemodialysis & 1.6 & 4.7 & $<.001$ \\
30-d mortality & 2.1 & 5.8 & $<.001$ \\
Operative mortality & 2.6 & 6.8 & $<.001$ \\
\hline Dapre
\end{tabular}

Data presented as median (25th, 75 th percentile) or \%. $M V$, Mitral valve; $M V R$, mitral valve replacement; $L O S$, length of stay. available cardiac surgery clinical databases, including the national STS ACSD, concerns the granularity that exists in the data regarding the etiology of MV disease. Without these details, it is difficult to completely appreciate which valves are appropriate for MV repair and which are amenable to MVR alone. This aspect of confounding certainly influences surgeon treatment allocation for MV disease. We are encouraged by the current steps taken by the STS to begin capturing more robust data related to MV etiology in its most recent data harvesting efforts, and we believe that future analyses will be able to better address these concerns when these data are available.

The principal findings of the present study concern the estimated associations observed between the average annual surgeon and hospital MV procedure volume and the likelihood for MV repair (vs MVR) after adjustment for the confounding influence of baseline patient risk. The independent examination of both hospital and surgeon procedure volume measures was considered, because both these metrics have been evaluated in previous investigations and have been advanced as potential quality performance measures for hospitals and surgeons performing MV operations by some investigators. ${ }^{5,7}$ As a result, these data demonstrated significant associations between each volume measure and the adjusted probability of $\mathrm{MV}$ repair compared with MVR. The strength of association, however, was much stronger and highly significant for the influence of the average annual surgeon volume than for the hospital volume, as indicated by their respective model likelihood ratios (59.89 vs 4.32 ). These 
observations have bolstered former analyses by Bolling and colleagues, ${ }^{7}$ who demonstrated that increased surgeon-level MV volume was independently associated with an increased propensity for MV repair, including estimates that surgeons performing $>100$ mitral cases annually were 3 times more likely to perform MV repair (vs MVR) than surgeons performing $<5 \mathrm{MV}$ cases annually (adjusted odds ratio, 3.78). ${ }^{7}$ The relationship between hospital procedure volume and the probability for MV repair was not nearly as robust after accounting for the influence of individual surgeon in the present analyses. Thus, it appeared that the hospital MV volume likely serves as a surrogate or proxy for the influence of surgeon volume and experience in relation to MV surgery.

The questions and implications of volume thresholds and the likelihood for the performance of either MV repair or MVR are one of ongoing investigation and debate. The findings in the present study have demonstrated that an inflection point appears at average annual surgeon volumes of approximately $\geq 20$ operations/y, at which the probability for MV repair increases dramatically (Figure 4, $A$ and $B$ ). From these empiric results, we subsequently compared the MV repair rates for categories of surgeons performing $<20$ mitral operations/y with those performing $\geq 20$ operations/y and found that the greater volume cohort of surgeons was 3 to 4 times more likely to perform MV repair (vs MVR) than surgeons performing $<20$ mitral operations/y. These data are complimentary to the few published reports that addressed the volume-outcome relationship that exists for the performance of MV repair. In the previously described work by Bolling and colleagues, ${ }^{7}$ an apparent threshold of approximately 40 to 50 mitral operations/y was noted at which the probability of MV repair became more constant at approximately $70 \%$. Other MV repair volume thresholds that have been advanced in the available studies included the recommendations by Bridgewater and colleagues ${ }^{10}$ for surgeon volumes of 25 repairs/y and hospital volumes of 50 repairs/y, which were arbitrarily defined thresholds extrapolated from volume-outcome relationships described in other surgical populations.

Unexpected in the present analyses was the observation that a small percentage of the lowest volume surgeons demonstrated an increased propensity to perform MV repair operations. Although definitive conclusions regarding these observations could not be made from these analyses alone, post hoc analyses demonstrated that the lowest volume surgeons with an average annual MV operation rates of 0 to 5 cases/y (18\% of surgeons) performed operations on highly selected patients with a low STS PROM (1.5\%) and exclusively moderate MR. It is also plausible that this subgroup of surgeons might represent young, relatively inexperienced surgeons who benefitted from the senior mentorship of more experienced, higher volume surgeons and influenced the choice for MV repair. Additional prospective investigation within the VCSQI organization is needed to draw more clear conclusions on these trends.

Critical to understanding the implications of the results of the present study are the risk-adjustment modeling techniques used to analyze the relationship between volume measures and the adjusted probability of MV repair. Although other reports have been published that used multivariable regression modeling for the investigation of the relationship between volume measures and the relative likelihood MV repair (vs MVR), the methods used in the present analysis were unique in that the present study did not focus on the contribution of other individual patient-related clinical characteristics and did not arbitrarily categorize the volume measures. The individual relationship of patient characteristics on the propensity for MV repair has been rather well established. ${ }^{5,7}$ In these analyses, the influence of patient-level risk was accounted for in aggregate using the widely accepted STS PROM score, yielding well-adjusted models for patient risk. More importantly, in the present analyses, we used restricted cubic spline transformations of each volume measure and the STS PROM variable to represent a more accurate and robust approach to modeling each continuous function. The techniques used in these analyses most accurately characterized the linear and nonlinear components that can exist in the relationship between hospital and surgeon volume and the likelihood of MV repair. ${ }^{13,14}$ An alternative approach used in previous cardiac surgical reports is the arbitrary categorization of volume measures into discrete categories (ie, quartiles, quintiles)..$^{5,7,10,11,15-17}$ Such an approach results in the conversion of continuous data (ie, surgeon volume) into categorical data, perhaps resulting in a crucial loss of information. ${ }^{13}$ The categorization of continuous volume measures is problematic because this technique discards information intrinsic to the original volume measure and arbitrarily selects cutpoints for defining categories and deriving conclusions. ${ }^{18}$ The ultimate result is a loss of explanatory power because the modeled effect of that variable is flat across the range of values included in that category. The results of the present series, therefore, serve to not only corroborate the volume-outcome association that exists between surgeon volume and likelihood for MV repair, but also to extend the examination of these results to provide a more precise description of the relationship of surgeon and hospital volume and the relative likelihood of performance of MV repair (vs MVR).

The reported findings have significant surgeon- and healthcare system-related implications. The present findings add to an increasing body of data addressing an apparent association between increasing surgeon procedure volume and the increased likelihood of MV repair compared with MVR. Our findings further promote the concept of the "referent surgeon" first forwarded by 
McCarthy. ${ }^{11}$ It is possible that referring cardiologists recognize centers of excellence and expert surgeons for MV repair and selectively refer candidates for MV repair to these centers and surgeons, and other valves deemed appropriate for MVR might be referred to surgeons and hospitals with lower MV repair rates. Recent trends in medicine and surgery have focused on risk-adjusted outcome reporting and the use of volume measures as a proxy for institution and provider quality. These results can be extrapolated to extend former recommendations by Gammie and colleagues $^{5}$ that performance of MV repair (vs MVR), especially for functional MR, should be considered a potential future performance measure within the cardiac surgical and cardiovascular medicine communities. ${ }^{5}$ Finally, these results highlight the critical importance of the role of the individual surgeon in the treatment allocation choice for the treatment of mitral disease. In the rapidly approaching era of percutaneous MV repair, surgeon volume and expertise as a gatekeeper should dictate access to this technology.

A fundamental challenge encountered in the present analysis, and one not addressed in previous STS ACSD analyses, is the lack of clinical and diagnostic data related to the valve morphology and etiology underlying MR. Thus, it is difficult in the current era to use multi-institution STS data registries to provide detailed estimates of which MVs remain candidates for MV repair and to match the comparisons in analyses. Current and ongoing efforts by the STS to expand the granularity of the collected data related to MV disease and operative characteristics will likely allow more robust analyses in the near future.

The limitations of the present study included the secondary analysis of a data registry and the observational nature of the VCSQI and STS ACSD. The study design was retrospective and subject to inherent selection bias. The reported results describe observed associations without assessing direct cause and effect relationships. The de-identified nature of the data further limited the ability to scrutinize hospital- and surgeon-specific details. The variance attributed to the unique characteristics of participating VCSQI hospitals could not fully be accounted for in the statistical analysis. In addition, the reported findings represent short-term, operative outcomes; intermediate- and longterm follow-up data were not available. Finally, the potential for unrecognized miscoding of data could have had an immeasurable effect on the reported results, which might have served to bias the results toward the null and further underestimated the magnitude of the association between volume measures and the modeled outcome, MV repair (vs MVR).

\section{CONCLUSIONS}

A significant variation in the performance of MV repair compared with MVR for moderate to severe MR persists in the modern surgical era. The average annual surgeon volume appeared more significantly associated with an increased likelihood for MV repair than institutional volume. An increased propensity for the performance of MV repair appears to exist for surgeons performing $>20$ MV procedures annually. These results could have important future implications on patient referral of care and quality measure assessment. In the upcoming era of percutaneous MV repair technology, surgeon volume and expertise as a gatekeeper should dictate access to this technology and the decisions for the best approach to MV repair.

\section{References}

1. Nishimura RA, Otto CM, Bonow RO, Carabello BA, Erwin JP, Guyton RA, et al 2014 AHA/ACC Guideline for the Management of Patients With Valvular Heart Disease: Executive Summary: A Report of the American College of Cardiology/American Heart Association Task Force on Practice Guidelines. J Am Coll Cardiol. 2014;63:2438-88.

2. Vahanian A, Alfieri O, Andreotti F, Antunes MJ, Barón-Esquivias G, Baumgartner H, et al. Guidelines on the management of valvular heart disease (version 2012): the Joint Task Force on the Management of Valvular Heart Disease of the European Society of Cardiology (ESC) and the European Association for Cardio-Thoracic Surgery (EACTS). Eur J Cardiothorac Surg. 2012;42:S1-44.

3. Bach DS, Awais M, Gurm HS, Kohnstamm S. Failure of guideline adherence for intervention in patients with severe mitral regurgitation. J Am Coll Cardiol. 2009; $54: 860-5$.

4. Toledano K, Rudski LG, Huynh T, Beique F, Sampalis J, Morin JF. Mitral regurgitation: determinants of referral for cardiac surgery by Canadian cardiologists. Can J Cardiol. 2007;23:209-14.

5. Gammie JS, Sheng S, Griffith BP, Peterson ED, Rankin JS, O’Brien SM, et al. Trends in mitral valve surgery in the United States: results from the Society of Thoracic Surgeons Adult Cardiac Surgery Database. Ann Thorac Surg. 2009; 87:1431-7; discussion 1437-9.

6. Acker MA, Parides MK, Perrault LP, Moskowitz AJ, Gelijns AC, Volsine P, et al. Mitral-valve repair versus replacement for severe ischemic mitral regurgitation. N Engl J Med. 2014;370:23-32.

7. Bolling SF, Li S, O’Brien SM, Brennan JM, Prager RL, Gammie JS. Predictors of mitral valve repair: clinical and surgeon factors. Ann Thorac Surg. 2010;90: 1904-11; discussion 1912.

8. Bolling SF. Can we predict mitral valve repair rates by individual surgeons mitral volume? Tex Heart Inst J. 2011;38:703-4.

9. Filardo G, Hamilton C, Hebeler RF Jr, Hamman B, Grayburn P. New-onset postoperative atrial fibrillation after isolated coronary artery bypass graft surgery and long-term survival. Circ Cardiovasc Qual Outcomes. 2009;2:164-9.

10. Bridgewater B, Hooper T, Munsch C, Hunter S, von Oppell U, Livesey S, et al. Mitral repair best practice: proposed standards. Heart. 2006;92:939-44.

11. McCarthy PM. When is your surgeon good enough? When do you need a "referent surgeon"? Curr Cardiol Rep. 2009;11:107-13.

12. Savage EB, Ferguson TB Jr, DiSesa VJ. Use of mitral valve repair: analysis of contemporary United States experience reported to the Society of Thoracic Surgeons National Cardiac Database. Ann Thorac Surg. 2003;75:820-5.

13. Greenland S. Dose-response and trend analysis in epidemiology: alternatives to categorical analysis. Epidemiology. 1995;6:356-65.

14. Kozower BD, Stukenborg GJ. The relationship between hospital lung cancer resection volume and patient mortality risk. Ann Surg. 2011;254:1032-7.

15. Puskas JD, Kilgo PD, Thourani VH, Lattouf OM, Chen E, Vega JD, et al. The Society of Thoracic Surgeons 30-day predicted risk of mortality score also predicts long-term survival. Ann Thorac Surg. 2012;93:26-33; discussion $33,25$.

16. Puskas JD, Thourani VH, Kilgo P, Cooper W, Vassiliades T, Vega JD, et al. Off-pump coronary artery bypass disproportionately benefits high-risk patients. Ann Thorac Surg. 2009;88:1142-7.

17. Qadir I, Salick MM, Perveen S, Sharif H. Mortality from isolated coronary bypass surgery: a comparison of the Society of Thoracic Surgeons and the EuroSCORE risk prediction algorithms. Interact Cardiovasc Thorac Surg. 2012;14: 258-62. 
18. Stukenborg GJ, Kilbridge KL, Wagner DP, Harrell FE Jr, Oliver MN, Lyman JA, et al. Present-at-admission diagnoses improve mortality risk adjustment and allow more accurate assessment of the relationship between volume of lung cancer operations and mortality risk. Surgery. 2005;138:498-507.

\section{Discussion}

Dr David A. Fullerton (Aurora, Colo). Thank you very much. Dr LaPar, I very much enjoyed your presentation and enjoyed reading your report even more. It is extremely well written, and it will be an excellent contribution to the data further defining the relationship of surgeon volume and hospital center volume in mitral valve surgical outcomes and the choice of operation.

I must confess that the sophisticated statistical method that you used is a little over my head. I do not question it. I just have to acknowledge up front that I do not fully understand it, and I suspect that many who read the report will likewise have similar questions. It is for that reason that I have a single question for you. You have done a very nice job of demonstrating a threshold of about 20 cases annually as being important in the surgeon's volume for defining, if you will, the percentage of mitral valvuloplasties that are performed in that surgeon's hands as opposed to replacements. Yet, as one looks at the final graphs that you displayed, one can see that even those surgeons who have very low volumes per annum have identical rates of repair as those that have very high rates or very high surgical volumes.

So I am curious as to how you might think this 20 cases per year might actually play a role, and how might you help explain, then, that even those centers and those surgeons with very low volumes actually had very high rates of repair?

Thank you very much, and I enjoyed your report.

Dr LaPar. Thank you very much, Dr Fullerton, for those questions and your kind comments.

The threshold that we observed of approximately 20 mitral operations per year and the relationship to performance of mitral repair is complimentary to several previous reports that have examined trends in mitral surgery.

We, too, were surprised by the findings that the probability of performing mitral operations was somewhat higher among very low-volume surgeons performing between 0 and 5 operations per year. We're not quite sure exactly what motivations account for this trend and unfortunately can't draw any definitive conclusions on this trend at this point without looking into the practice patterns of these hospitals a little more closely.

When we looked into the data available to us for this study for this subgroup of patients, we did notice some interesting trends that are somewhat descriptive. We found that these patients had very low predicted risk of mortalities-less than $1.2 \%$ for most of them. We also found that these patients tended to have almost exclusively moderate mitral regurgitation.

These findings may also represent the experience of relatively young surgeons receiving senior mentorship for mitral repair at some centers, but, because of the de-identified nature of this data, this is truly speculative at this point.

Dr Lawrence H. Cohn (Boston, Mass). I just want to ask you an obvious question. Is this type of study going to lead to the state of Virginia dictating who and who will not be able to perform mitral valve surgery in the future?
Dr LaPar. That is certainly is an important question. I don't think it's going to have too many direct policy implications regarding treatment allocation in the state of Virginia, but I think it does generally support what we've seen in the national data: That surgeons who have expertise in certain areas are more likely going to perform mitral repairs where appropriate.

Dr W. Randolph Chitwood (Greenville, NC). Damien, I thought this was a great study, and it sort of parallels some of the work that Jim Gammie did in the past. But the 1 point it does not state, and neither does Jim Gammie's report or any of these reports, is what about the repairable valves? In other words, repairable versus rheumatic versus, let us say, you are in a small hospital and you have 15 cases coming in there, are these all posterior leaflet P2 resections, which are fairly straightforward and most can be repaired, or are they anterior leaflet resections?

So I think we must sometime study the data in our databases in depth to know exactly what we are repairing and what we are not. Because I would expect if you take the low-volume surgeons and the low-volume centers with good results, that they are being smart enough to take on the straightforward posterior leaflet cases, which are $80 \%$ of the degenerative disease cases, and what about those with rheumatic valves?

So your database, I do not believe separates this nor does our national database. Thus, this is a cry, wouldn't you say, especially when Dave Adams presented a report yesterday about guidelines and items of this nature. Because I guarantee you the members of this organization, the European Association for Cardio-Thoracic Surgery, and the STS would be up in arms if someone mandated that one cannot repair a MV unless you have done $25 \mathrm{MV}$ repairs because some people do achieve excellent results. So, comment on the pathophysiology.

Dr LaPar. I couldn't agree more with you that a fundamental limitation of a regional dataset like this, but also within the STS national data, is a lack of information related to mitral valve etiology and pathology. And, as you are aware, it is something that the STS is really trying to improve in the new data-harvesting efforts.

Unfortunately, in this data, we can't tease that out either in this data because this is an analysis of a regional STS database.

Dr Chitwood. The sin is in your propensity matched patients, only $50 \%$ were repaired. The European data show more. But still, if you are considering repairable valves, almost $100 \%$ should be repaired.

Dr Daniel H. Drake (Traverse City, Mich). I direct the Michigan Society of Thoracic and Cardiovascular Surgeons Mitral Initiative. I enjoyed your presentation immensely.

In Michigan, we have started to see internal referrals between Society surgeons. The complexity of repair can be predicted from the diagnostic transesophageal echocardiogram. After a review of the transesophageal echocardiogram (which is performed well in advance of surgery), and depending on the individual surgeon's level of comfort and expertise, we have occasionally seen referrals between surgeons. This was somewhat unanticipated and is an interesting process to observe. Are you seeing similar referrals between surgeons? Also, what are your efforts to predict complexity?

Dr LaPar. I am sorry, what was the last part?

Dr Drake. What are your efforts toward predicting the complexity of repair? 
Dr LaPar. You question is quite interesting because we haven't been able to empirically examine the trends within our organization for internal referrals.

Dr Drake. How about the discussion during your collaborative meetings? Do the surgeons seem at all interested in referral among themselves?
Dr LaPar. The VCSQI certainly is interested in developing processes of care that result in the delivery of the best patient care. Although we haven't directly discussed the trends of internal referrals for mitral repair, this is certainly something that may look into further in future collaborations.

Dr Drake. Thank you. 